\title{
HIGH INTENSITY PHENOMENA OBSERVED IN THE CPS
}

\author{
Y. Baconnier, J. Gareyte, P. Lefèvre \\ CERN, Geneva, Switzerland
}

\section{Summary}

A review of high intensity phenomena observed in the CPS is presented. At injection an attempt is made to describe the combined effect of space charge and resonances, which result in a 10 ss of $70 \%$ of the injected beam intensity and a reduction by a factor of 3 of the central core brilliance. Results of measurements on the effect of a localised bad vacuum are given. The situation at transition is briefly described.

Seven different instabilities observed in the CPS in longitudinal or transverse phase plane are analysed. The compensation techniques are described in all cases. Whenever possible an attempt has been made to give some qualitative explanation of the mechanism.

\section{Introduction}

Last year a new customer, the ISR, was added to the 1 ist of the CPS users. In the years to come we will have to accept particles from the $800 \mathrm{MeV}$ Booster and to inject our beam in the $300 \mathrm{GeV}$ machine. As an injector both for the ISR and now the $300 \mathrm{GeV}$ machine the CPS should not only be a high intensity accelerator but also should deliver a beam as dense as possible. Consequently a high priority is given in the CPS to the study of high intensity phenomena. This review being made while the work is still in progress, we have tried to 1 ist not only the effects we understand but also those for which both experiment and theory are uncomplete.

\section{$50 \mathrm{MeV}$ Injection}

About a year ago we modified our injection and trapping situation in the CPS in order to increase the final intensity of our machine. We were previously using the "high trapping" technique, described several years agn ${ }^{1}$, combined with single turn injection, and a field rate of rise (B) of about 1.3 tesla per second. This technique had the inconvenience of reducing the longitudinal acceptance. Also, during the process, one had a rather strongly bunched beam and therefore important space charge effects. On the other hand the large $\dot{B}$ provided a faster crossing of resonances, space charge effects could only develop for a short time.

We now use multiturn injection combined with $R F$ adiabatic trapping ${ }^{2,3}$. A reduction of $\dot{B}$ is necessary in order to achieve a good longitudinal adiabatic trapping. The new value of $\dot{\mathrm{B}}$ is 0.35 tes $1 \mathrm{a} \mathrm{s} \mathrm{s}^{-1}$ for $80 \mathrm{~ms}$ then we increase it to about 1.9 tesla $s^{-1}$ for the rest of the acceleration. The maximum longitudinal density of particles is reduced by a factor 2 to 3 but we stay longer on resonances at low energy. This last point turned out to be of some advantage because we could then separate the various resonances and therefore $\mathrm{f}$ ind a better compensation.

In order to achieve a high intensity we had to modify to a large extent the transverse focusing in the CPS. The injection quadrupoles, DC powered, bring the working point for small intensity above $\mathrm{Q}_{\mathrm{V}}=6.5$, that is on the other side of the $\frac{1}{2}$ integer stop-band that we have to cross when we accelerate a small beam.
In fact, as shown in Fig. 1, we cross several stop-bands

The working point in the diamond time and space charge included
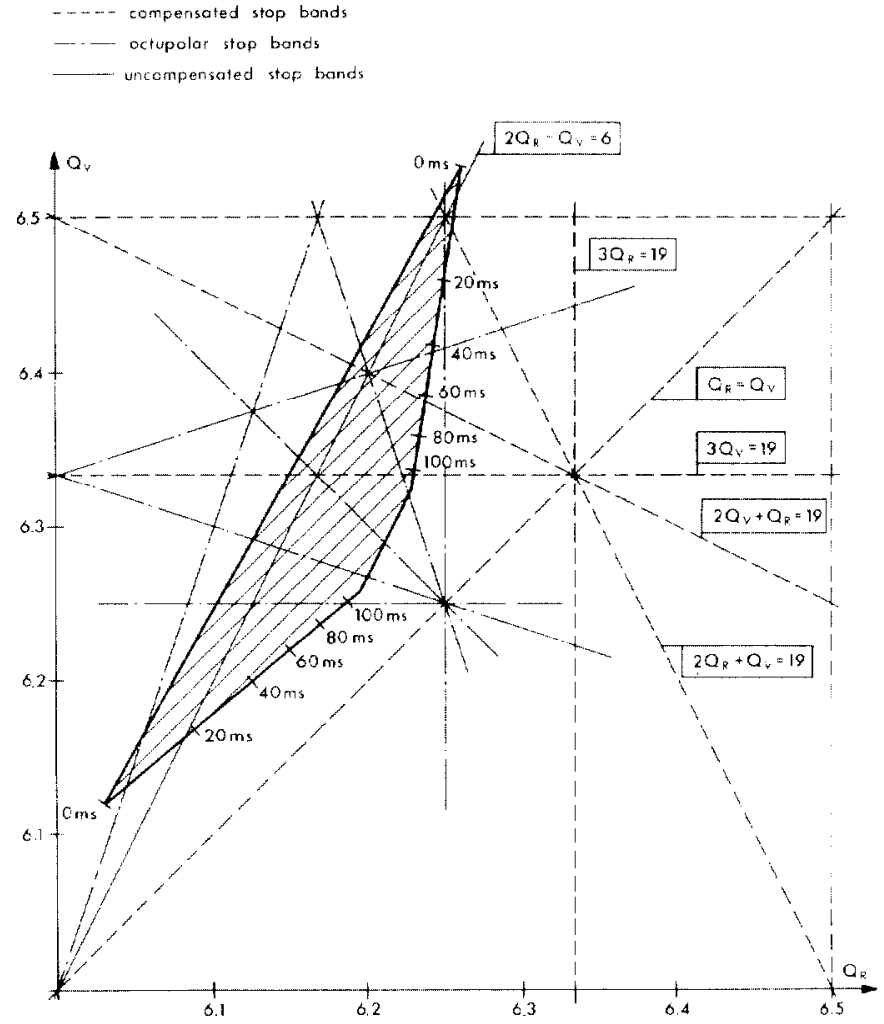

with the low intensity beam ${ }^{4}$, our working conditions are clearly not adapted to such a beam.

At its best our linac produces ${ }^{5}$ a beam of $110 \mathrm{mr}$, that is $1.410^{13}$ protons in the 20l.s of our 3 turn-in. fection. About $50 \%$ of these are lost in those $3 \mathrm{first}$ turns so that the maximum number of injected protons never reaches more than $7.10^{12}$. The maximum number of protons we are able to accelerate to top energy being about 2.1012, our trapping efficiency is only about $30 \%$, the overall transfer efficiency being of the order of 15\%. Must of these losses lake place in the first $10 \mathrm{~ms}$ of the PS cycle.

I. one assumes that the CPS beam after $10 \mathrm{~ms}$ is roughly gaussian and fills the vacuum chamber one finds that the corresponding brilliance if 20 times smaller than what it should be in an ideal transfer. whis indicates that in addition to the $85 \%$ intensity loss we lose a factor of 3 in brilliance.

Thus, keeping the sare assurptions one can calmulate the maximum single particle $Q$ shift inside the beam:

$$
\begin{aligned}
& \Delta Q_{\mathrm{V}}=0.4 \\
& \Delta \mathrm{Q}_{\mathrm{R}}=0.2
\end{aligned}
$$

These $Q$ shifts indicated in Fig, 1 seen the reasonable numbers to quote if one assumes that the distributions are stable. 
Experimentally one can verify that the high intensity beam is not sensitive to the perturbations exciting the stop-bands $2 Q_{V}=13$ and $2 Q_{V}+Q_{R}=19$, but starts being sensitive to $3 Q \mathrm{~V}=19$; this last stop-band is excited by sextupoles which are not symmetrical with respect to the horizontal plane. Also one should mention that most of our recent intensity increase resulted from sextupolar stop-band compensation.

After those very approximate estimations several questions remain

- Are the distributions stable?

- Why cannot we move further the zero space charge working point?

- Is the beam really filling the vacuum chamber after $10 \mathrm{~ms}$ ?

or to summarize : what happens during the first 10 ms ?

We believe that if we want to make further progress we have to study the dynamical properties of particle distributions. On the experimental side we are testing a computer acquisition of the Ionic Beam Scanner signals which we hope will help us to progress in that direction.

\section{Vacuum Effect}

About 2 years ago we decided to improve by an order of magnitude the vacuum in the CPS. An experiment was set up in order to prepare this decision. We installed nitrogen cooled baffles ${ }^{6}$ around the vacuum chamber in most of the straight sections; this way we could reduce the pressure from $2.110^{-6}$ to $1.210^{-6}$ torr for a few hours. In this limited amount of time we were able to raise the maximum circulating beam intensity by about $10 \%$ which was already a favorable indication.

During the same experiment we had prepared a localised adjustable leak. The effects were much more evident even though the average pressure never reached the previous value of $2 \cdot 10^{-6}$

The vertical "Head-Tail" instability appeared very quickly at high energy, we could stabilise it by increasing the energy spread in the beam.

Close to injection important beam losses appeared at the time we were crossing sextupolar stop-bands. We could eliminate most of these losscs by installing a sextupolar compensation.

Finally we tried two gases Ke and Air. The effects were similar for two different pressures (localised on about $5 \mathrm{~m}$ )

$$
\begin{aligned}
& 7.10^{-5} \text { torr for Xe } \\
& 2.10^{-4} \text { torr for Air. }
\end{aligned}
$$

These correspond to the same reading of the ionisation gauge. These effects seem therefore to be related to the number of ionisable electrons. We have not yet found a satisfactory explanation.

\section{Transition}

For the same reasons that make it an interesting injector for ISR, namely a high longitudinal density, the CPS proved to be a difficult machine from transition point cf view. It has strong longitudinal space charge effects. Picture 1 shows how the maximum longitudinal density (roughly the inverse of bunch length) varies when crossing transition in an uncompensated way. Linear theory of longitudinal space charge explains the oscillations but not the drop in average density.

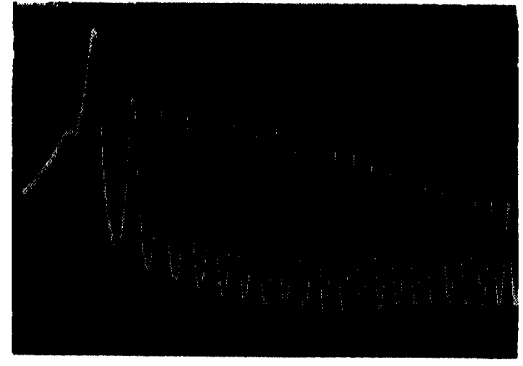

Pict. 1 Transition without Q-Jump

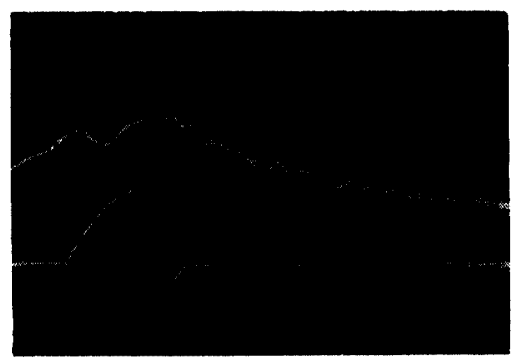

Pict. 2 Transition with Q-Iump

- Longitudinal density

- Current in the quadrupoles

Transverse space charge and non-linear longitudinal space charge might explain this drop, calculations of these effects are underway but no result is yet available.

In 1969 the Q-jump technique was tried successful$1 y$ on the CPS giving results shown on Picture 2. More recently a more powerful method called $\gamma_{t r}$-jump ${ }^{8}$ was investigated but not tried experimentally. The idea of both these techniques is to achieve a long equilibrium bunch length by a large and fast decrease of $\gamma_{t r}$ just after transition crossing; in the $y_{t r}$-jump the change in $\gamma_{t r}$ can be made significantly larger than the change of $Q$ by exciting two sets of quadrupoles with opposite polarity. Various techniques are available to match the bunches to their lengthened equilibrium situations. We hope with this method to be able to cross transition with $10^{13}$ particles.

\section{Instabilities}

The number of collective phenomena observed ${ }^{9}$ has steadily increased with the intensity of our machine. The easiest to recognize are the instabilities but various emittance blow-up ${ }^{10}$, that we measure, could have their origin in similar effects.

\section{Longitudinal Instabilities}

Rebunching : for slow ejection or target operation we debunch the beam in order to deliver a structurefree spill to the physicists. After this debunching operation we noticed (with the help of the experimenters !) that the beam was rebunching on a 5 th harmonic cf cevolution frequency. Hereward showed ${ }^{11}$ that the impedance of the cavities, tuned at injection frequency during this operation was responsible for this effect. He also proposed various cures. The stagger tuning of the cavities was tried successfully. For the improved CPS beam we will have new RF cavities, a short circuit of the gap during debunching operation has been foreseen. 
"Hereward Damping": that is the name we give to a damping system for coherent bunch shape oscillations of the type H.G.Hereward ${ }^{12}$ and E.C.Raka ${ }^{13}$ described a few years ago. The source of the oscillations we observe is not known but one supposes that they have their origin in imperfections of the beam control ${ }^{14}$. Picture 3 shows the envelope of the signal obtained from a sum pick-up electrode (roughly the inverse of bunch length). With the damping system Picture 4 the oscillations have disappeared. The oscillations induced at transition are damped.

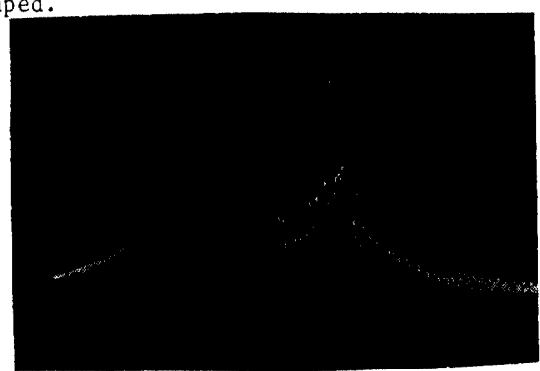

Pict. 3 "Hereward Damping" off. Longitudinal density oscillations.

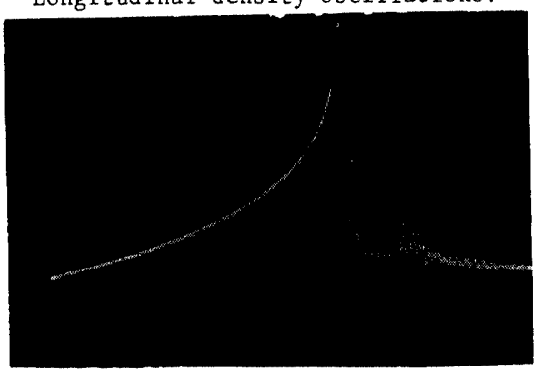

Pict. 4 "Hereward Damping" on. Longitudinal density oscillations.

Dipole Longitudinal Instabilities 15 : more recently an instability of the bunch position developped for the short bunches we have if we go through transition with Q-jump. A similar instability was observed and cured at the AGS ${ }^{15}$. Picture 5 shows a mountain range display of the position of one bunch with respect to the RF sinewave triggering the scope. The bunches oscillate with different phases with respect to the RF in a pattern of 5 wavelength around the machine. This corresponds to a $\pi / 2$ phase shift from one bunch to the next which is the most unstable mode in our case. The beam control, seeing an average position of the bunches, is not effective.

In the CPS it is a parasitic resonance in the accelerating cavities which is responsible for the instability. Each bunch leaves in the cavity a wake that will excite the next bunch. If we do not use Q-jump at transition, the bunches are longer so that the instability is Landau damped by RF non-1inearities.

We succeeded in damping this instability by powering at half RF frequency one of our cavities. This provides a bunch to bunch synchrotron frequency spread which decouples the bunches. We did this after discovering that we had to select a special pattern of synchrctron frequency modulation to be efficient. For example, a modulation at $\frac{1}{4}$ of the RF frequency would not be efficient. This effect was explained by including the beam control in our model. We can understand it by comparing the two types of modulation pattern. If we note + a bunch that has a higher synchrotron frequency and - the opposite one has the following patterns for the two modulations : a) $\frac{1}{2} \mathrm{RF}$ frequency $:+-+\cdots+\ldots$

b) $\frac{1}{4}$ RF frequency : $+0-0+\ldots \ldots$

If we represent on one phase space diagram four consecutive bunches oscillating at $\pi / 2$ phase shift one from the other we obtain Fig. 2 where the arrows indicate the phase drift corresponding to the frequency modulation. In case a) the center of gravity of the four bunches does not drift. In case b) the center of gravity does move. The beam control will counteract the motion, therefore diminishing the effect of the modulation.

It is by a similar interaction with the beam control that we could also explain the fact that the instability developped even in the case where several bunches were missing, for example, after fast extraction of a few bunches.

This stabilisation however is not powerful enough. When half of the available power at $\omega_{\mathrm{rf}} / 2$ is applied (Picture 5) the bunches still oscillate. At full power the stabilisation is barely sufficient. We cannot increase the number of cavities that we use for this purpose. For these reasons we are now looking into an active feed back, but with the large harmonic number of the CPS $(h=20)$ one has to be careful not to excite other modes.
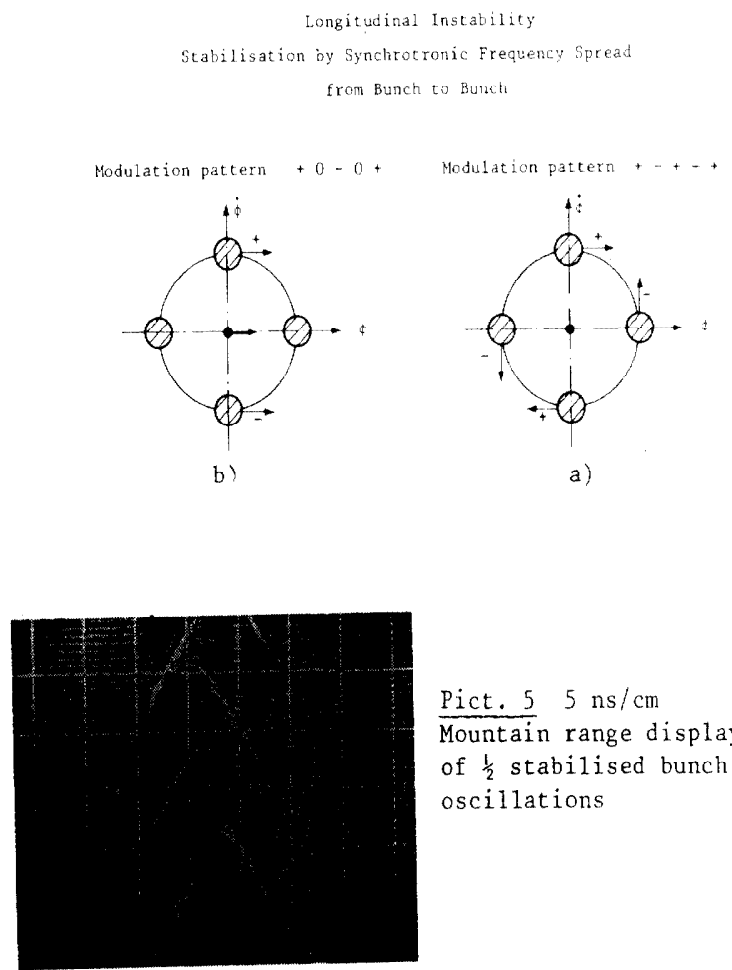

Pict. $5 \mathrm{~ns} / \mathrm{cm}$ Mountain range display of $\frac{1}{2}$ stabilised bunch oscillations

Pict. $65 \mathrm{~ns} / \mathrm{cm}$ Mountain range display of non stabilised bunch oscillations

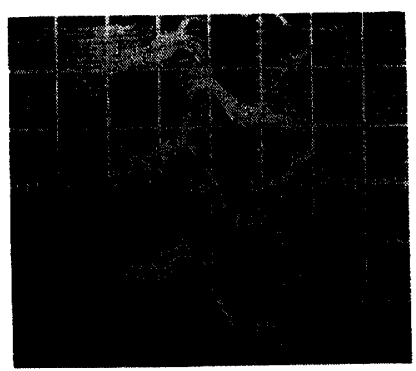


Multipole Longitudinal Instability : Picture 6 corresponds to the same situation as picture 5 but without the stabilisation. Clearly at large amplitudes the oscillations are not purely dipole. One could think that the filamentation explains the difference but the experimental evidence is that this is probably not the only effect. In fact, we were able to calculate 16 e-folding times of about $200 \mathrm{~ms}$ for a bunch shape nscillation with a similar mechanism. The frequency modulation seems rather efficient in stabilising these multipole instabilities. In case an active feed-back would work on the dipole instability we might still have to keep the modulation technique for the multipole effects.

With the high intensity beam $\left(10^{13}\right.$ p.p.p) the bunches will be longer, we will have new cavities. Predictions are therefure difficult to make.

\section{Transverse Instabilities}

Ionic 0scillations : this was the first instability to be discovered, explained and suppressed in the CPS. It is a bunch to bunch coherent radial instability which starts at low energy. Picture 7 shows the voltage induced by the beam in the sum and radial electrodes, one sees the bunches oscillating with a well defined mode : with a pattern of 6 wavelength around the machine and a local frequency $(Q-6)=0.25$ per turn visible on Picture 7.H.G.Hereward found ${ }^{17}$ that the source of the instability is the ionic wake that protons leave behind them, the necessary phase shift between force and motion being introduced by the relative motion of the proton bean and the ion cloud. The final cure came with the improvement of the vacuum.

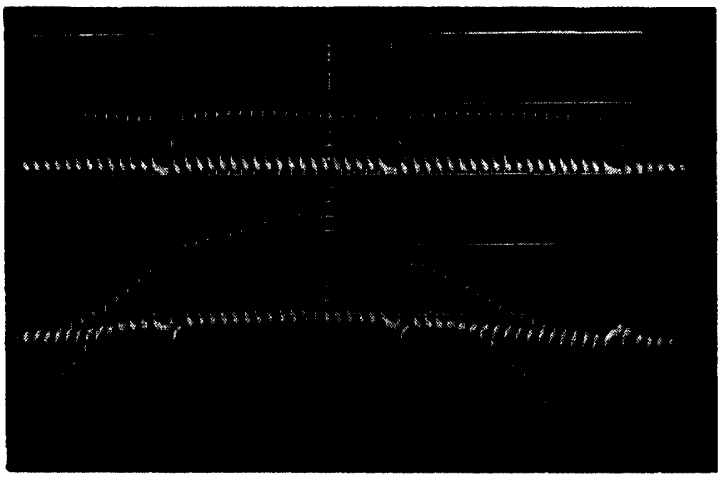

Pict. 7 Ionic 0scillations

- Sum signal from pick-up electrodes

- Radial signal from pick-up electrodes

$6 \mathrm{GeV} / \mathrm{c}$ Vertical Instability : this rather long name implies already that we do not understand this instability. In fact we can hardly see it. Two effects are visible : - we lose beam if we do not stabilise it by octupoles - the Ionic Beam Scanner, Picture 8 , shows that the beam vertical size is increasing with a growth rate of about 10 to $20 \mathrm{~ms}$. We do not see anything on the vertical pick-up electrodes. We have only observed this instability from 6 to $9 \mathrm{GeV} / \mathrm{c}$ when we go through transition using the Q-jump technique that is when we have the shorter bunches. At first it would look like a quadrupole type motion of the bunch. However a normal head-tail motion with a high mode number would not be visible through our $35 \mathrm{MHz}$ 1 imited band-width pick-up stations, We can avoid the losses with relatively low current in our octupoles, but we have to feed them with all the power we have to reduce the beam size at top energy. In other words the octupoles stop the growth at a certain amplitude.
This instability has been discovered fairly recently, a preliminary investigation 18 of wall effect does not give an explanation of such fast growth rates. We are improving the pick-up electrodes and should in principle get better observations soon.

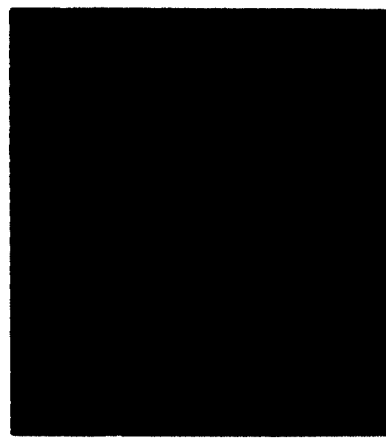

Pict. 8 Mountain range display $(5 \mathrm{~ms} / \mathrm{cm})$ of the Ionic Beam Scanner signal during the $6 \mathrm{GeV} / \mathrm{c}$ vertical instability.

Vertical Head Tail : this instability we call head tail by analogy with the instability described for Adone ${ }^{19}$. It appears around $10 \mathrm{GeV} / \mathrm{C}$ and we usually stabilise it with octupoles. On Picture 9 the vertical pick-up electrode signals induced by 5 bunches during a few thousand turns are superimposed.only one bunch oscillates : it is a single bunch instability. The signals from the oscillating bunch are only twice the signals from the non oscillating one, even though we were losing beam during this time. This cotses from the fact that the frequency in the bunch is outside the bandwidth of the pick-up station (probably mode 2 in terms of head tail modes). Picture 10 shows the circulating beam intensity (we lose beam) and the detected signal from the vertical pick-up : the e-folding time is about $10 \mathrm{~ms}$. Picture 11 is an lonic Beam Scanner picture of the phenomenon, similar to the signal we obtained for the $6 \mathrm{GeV} / \mathrm{c}$ instability.

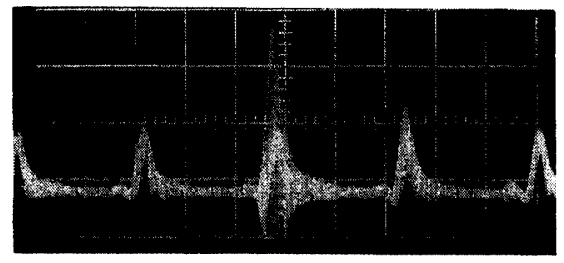

Pict. 9 Head Tail : a single bunch instability

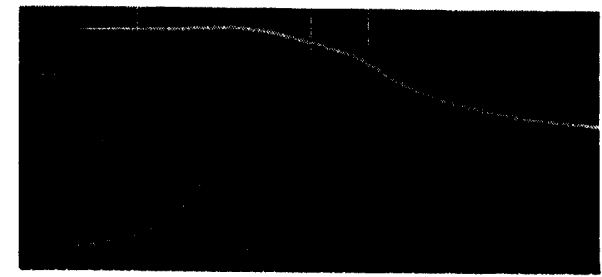

Pict. 10 Head Tail Instability. $10 \mathrm{~ms} / \mathrm{cm}$ - circulating beam intensity

- detected signal from vertical pick-up electrode

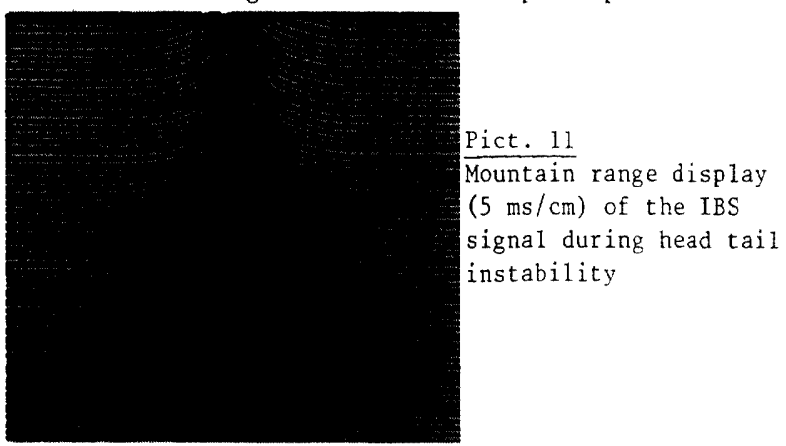


Several difficulties appear if we want to compare our measurements with the available theory of head tail:

The betatron frequency spread in the beam is much stronger than in an electron machine. Even the calculation 20 that zotter did for the ISR does not cover all the situations where we have observed instability (we can vary tie $\mathrm{dQ}_{\mathrm{V}}$ /dE in our machine by using sextupoles). In other words the betatron phase shift due to energy oscillation for a particle going from the head to the tail of the bunch is from 5 to 30 radians while in Sands model it is supposed to be of the order or less than a radian. Although zotter extended this calculation to large phase shift one can doubt that the model of a hollow bunch remains valid.

We observe e-folding times as low as 2 or 3 synchrotmic periods. This implies rather power forces that we have not been able to find, if one still apply the theory as modified by zotter the growth rates for wall effect are an order of magnitude too small.

We have not yet been able to find a mechanisrn which would explain the strong influence of a local bad vacuum as al ready mentioned.

Following a remark by $F$. Sacherer we calculated that the space charge detuning at $10 \mathrm{GeV} / \mathrm{c}$ is about as big as the $Q$ shift due to energy oscillations. In fact below $10 \mathrm{GeV} / \mathrm{c}$ the space charge detuning is larger and above it $i_{\mathrm{E}}$ smaller.

\section{Conclusion}

At injection the situation is still unclear but the more intense $800 \mathrm{MeV}$ heam that the new injector will deliver should be less uncomfortable in our machine from the point of view of space charge, than our present $50 \mathrm{MeV}$ beam. At transition we need better understanding but the technique of $\gamma t r^{-j u m p ~ s h o u l d ~ h o p e-~}$ fully solve our problems. We have observed and more or less stabilised a total of seven different instabilities but we still have some dilution during the acceleration. The fact that in the near future the CPS will be at the centre of a complex of 4 machines makes it all the more important to solve our problems.

\section{Acknowledgements}

The collaboration of a variety of people is necessary to achieve any improvement in this field. It mixes theory, experimentation, new instrumentation and new hardware. We therefore acknowledge the work of many people in the MPS Division and the constant support of P.H. Standley, H.G. Hereward has been at the origin of most of the progresses which were made. We thank him fur the long discussions we had concerning this review. We also profited from discussions with D. Boussard, M. Bouthêon, W. Fardt and A. Sqrenssen.

\section{References}

1. K.Johnsen. High trapping efficiency in synchrotrons with phase lock. 1961 Intern. Conf, on High Energy Accelerators, p. 194.
2. M.Bouthéon, J.Gareyte, E.Schulte. Mise au point d'un cycle d'accélération utilisant la capture adiabatique dans le PS. CERN/MPS/CO 70-2.

3. M.Bouthêon, M.Weiss. Note concernant 1'injection dans le PS à $50 \mathrm{MeV}$. CERN/MPS/CO/LIN 70-1.

4. Y.Baconnier, M.Bouthên, P.Lefèvre. Measurements on the $50 \mathrm{MeV}$ injection. To be published.

5. P.Têtu. Notes d'information sur le faisceau et les mesures de faisceau du Linac. MPS/LIN-Notes 70-7.

6. P.Riboni. Private communisation. See also

P.Coet, P.Riboni. The PS inproved vacuun system. MPS/ML 70-2.

7. W. Hardt, G.Mer1e, D.Möh1, A.Sфrenssen, L.Thorndahl. Experimental verification of the $Q$-jump method for passing transition with dense beams. 7 th Intern, Conf. on High Energy Accelerators, Yerevan 1969.

8. L.C.Teng. Compensation of space charge mismatch at transition. NAL FN-207/0/00 April 1970.

9. J.Gareyte. Observations récentes des instabilités dans le PS. MPS/DL - Note 70-6.

10. E. Brouzet, C.Johnson, P.Lefèvre. Mesures des dimensions verticales du faisceau du PS. MPS/DL Note $70-21$.

11. H.G.Hereward. Effects of cavities on debunching. Rough estimates for the CPS. CERN/MPS/DL 69-7.

12. H.G.Hereward. Second order effects in beam control system of particle accelerators. 1961 Intern. Conf, on High Energy Accelerators, p. 222.

13. E. C. Raka. Damping bunch shape osciliations in the AGS, IEEE Trans. Nuc. Sci. NS-16, No. 3, 182-6.

14. D.Boussard. MD Nole on "Hereward Damping". MPS/SR/MD 70-1.

15. M.Q.Barton, E.C.Raka. Beam loading instabilities in the RF system of the Brookhaven AGS. USSR 2 nd Nat. Conf. on Particle Accelerators.

Y.Baconnier, D.Boussard, J.Gareyte. Some preliminary results on coherent longitudinal instabilities in the CPS, CERN/MPS/SR 70-6.

D.Boussard, J.Gareyte and D.Möhl. Compensation of longitudinal instability in the CPS. CERN/MPS/ SR 70-8.

D.Mohl. Bunch to bunch frequency spread to stabilise coherent oscillations. CERN/MPS/DL 70-9.

1). Boussard, J.Gareyte, D.Möh1. The missing bunch experiment. MPS/DL - Note 70-34.

J.Gareyte. Landau damping factors for the longitudinal instability in the CPS. CERN/MPS/DL 71-2.

D.Boussard, J.Gareyte, D.Möh1. Study and compensaLion of colerent longitudinal instability in the CPS. This Conference.

16. Y, Baconnier. Bunch shape instability induced by a cavity. CERN/MPS/DL 70-15.

17. H.G.Hereward. The instability of radial betatron oscillations in the CPS. MPS/Int. DL $6 / 1-8$.

18. D.Möh1. Intensity limitations in the present and future PS. To be published.

19. C.Pellegrini. On a new instability in electron positron storage rings (the head tail effect). LNF 69-45. Laboratori Nationali di Frascati.

M.Sands. The head tail effect . An instability mechanism in storage rings. SLAC-TN-69-8.

20. B.zotter. The head tail effect in the ISR. CERN/ISR/TH 69-60. 